\title{
Structure-Function Interactions in the Therapeutic Response of Diabetic Neuropathy
}

Anders A. F. Sima

\begin{abstract}
Conventional electrophysiologic measurements such as nerve conduction velocity, compound action potential, and neuropathic symptom scores have been used to assess the severity of diabetic neuropathy and have been the major efficacy end points following pharmacologic intervention trials. Unfortunately, these measurements are not particularly sensitive and their reproducibility is, at best, good. Detailed morphometric analysis of sural nerve biopsies has evolved as a sensitive and highly reproducible method for assessing the severity and progression of diabetic peripheral neuropathy. In this study we demonstrate highly significant correlations between morphometric

parameters of diabetic sural nerves and electrophysiologic and sensory score measurements of the same sural nerve. These data suggest that detailed morphometric examination is a more sensitive and reproducible method for assessing the severity of diabetic neuropathy, and that quantitative morphometric parameters provide sensitive indicators of electrophysiologic and clinically meaningful nerve damage. Morphometric analysis of sural nerve biopsies therefore constitutes a rational basis for sensitive efficacy end points in the design of future therapeutic clinical trials. (Journal of Diabetes and Its Complications 6;1:64-68, 1992.)
\end{abstract}

\section{INTRODUCTION}

A number of methods are available for evaluating diabetic neuropathy; however, each is associated with some limitation. Electrophysiologic parameters such as nerve conduction velocity and clinical assessments such as neuropathic symptom scores are valuable for the clinical assessment of diabetic polyneuropathy. Unfortunately, these techniques are not reliable indicators of the severity of diabetic polyneuropathy, nor are they highly reproducible. In recent years, sural

Reprint requests to be sent to: Dr. Anders A. F. Sima, University of Michigan, 1150 West Medical Center Drive, Diabetes Research and Training Center, Ann Arbor, MI 48109, USA.

Michigan Diabetes Research and Training Center, University of Michigan, Ann Arbor, Michigan, USA nerve biopsy has been utilized as a research tool to evaluate structural changes taking place in diabetic neuropathic tissue. ${ }^{1}$ Morphometric analysis of sural nerve biopsies has developed as a sensitive and reproducible technique for determining the severity and progression of diabetic neuropathy. On the other hand, widespread use of nerve biopsy is not practical for assessing clinical responses in large populations of patients. Nevertheless, if the results from morphometric analysis of disease activity could be correlated with electrophysiologic and clinical parameters, then morphometric parameters might be used to evaluate therapeutic responses in clinical trials.

This paper will summarize data that were obtained recently in clinical diabetic neuropathy research. First, the correlation between various structural abnormalities in clinically overt diabetic neuropathy in humans 
and electrophysiologic parameters will be examined. Second, the correlation between specific structural abnormalities and sensory scores taken from the same sural nerves will be discussed.

\section{CORRELATION BETWEEN STRUCTURAL AND ELECTROPHYSIOLOGIC ABNORMALITIES IN DIABETIC NEUROPATHY}

In clinically overt diabetic neuropathy there is a loss of functional units such as myelinated nerve fibers. Moreover, preserved fibers in the diabetic nerve are smaller in size, as compared with age-matched controls. In addition, specific structural lesions occur at various sites in these functional units, particularly at sites that are of major functional importance. Lesions that occur in the chronic diabetic nerve consist of nerve fiber loss, atrophy, and specific fiber lesions, and may be termed the "triopathy of diabetic neuropathy."

The nerve fiber population of peripheral nerve constitutes the structural substrate for nerve function. In the myelinated nerve fiber the electrical impulse is generated at the node of Ranvier and propagated along the internodal axon. Hence, the maintenance of normal nerve conduction velocity is dependent on the functional and structural integrity of the node of Ranvier and the internodal segment.

The characteristic structural abnormalities occurring in diabetic nerve affect the node of Ranvier and the internodal axon. These progressive, structural lesions are therefore likely candidates for causing the impaired function of the chronically diabetic nerve.

The node of Ranvier of myelinated fibers is the site of a sequence of structurally well defined changes, both in murine and human diabetic neuropathy. These changes are initiated by nodal and paranodal swelling, and abnormality which has been extensively studied in the diabetic Bio-breeding Wistar (BB/W) rat and subsequently identified in the streptozotocin diabetic rat. In human diabetic subjects, nodal swelling appears to be a consequence of intra-axonal sodium accumulation. ${ }^{2}$ Nodal swelling is followed by the progressive loss of axo-glial junctions, leading to detachment of the terminal myelin loops from the paranodal axolemma, which in turn results in paranodal demyelination and widening of the nodal gap. Paranodal demyelination is repaired by the formation of new myelin, resulting in intercalated, thinly myelinated nodes.

We previously discussed the electrical events which occur at the node of Ranvier and how these events relate to various structural lesions. ${ }^{3,4}$ In a normal situation, the terminal myelin loops are anchored to the axolemma by so-called axo-glial junctions (Figure 1). The axo-glial junctions form a barrier system, preventing lateral migrations of the nodal sodium channels

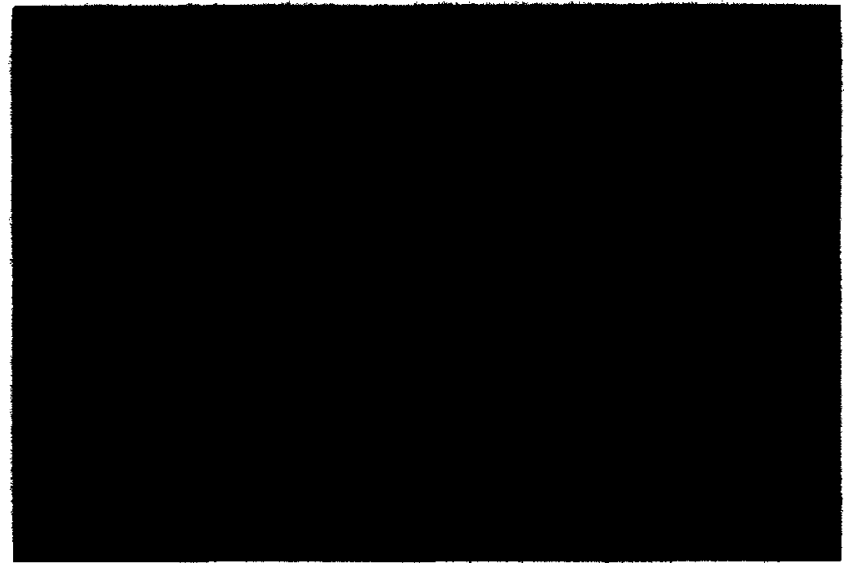

FIGURE 1 Schematic illustration of the nodal changes occurring in diabetes (right) compared to the normal situation (left). Note loss of axo-glial junctions (red original figure) in the diabetic nerve permitting migration of sodium channels (yellow original figure) from the nodal axolemma into the internode (arrows).

away from the nodal axolemma. One of the characteristic lesions that is seen in the $\mathrm{BB} / \mathrm{W}$ and streptozotocin diabetic rats and in human diabetic neuropathy is a progressive loss of these junctional complexes. ${ }^{2}$ The progressive loss of axo-glial junctions allows for the migration of sodium channels into the internodal axolemma. The diminution of the density of sodium channels at the node of Ranvier correlates with a decrease in sodium permeability that has been demonstrated experimentally, and is associated with a delay in the generation of the electrical impulse at the node of Ranvier.

With respect to internodal abnormalities, we and others have demonstrated axonal atrophy as being a characteristic lesion in both experimental and human diabetic neuropathy. Axonal atrophy is followed by axonal degeneration and Wallerian degeneration, leading to a net loss of functional nerve units in the diabetic nerve. The fiber atrophy that is observed is caused mainly by atrophy of the axon, associated with a corrugated and wrinkled appearance of the surrounding myelin sheath, which is relatively unaffected by the disease (Figure 2).

These axonal abnormalities can be quantified in detail. One method of quantifying axonal atrophy is the measurement of the axon/myelin ratio. The axon/myelin ratio is obtained by measuring the cross-sectional area of the axon and dividing it by the thickness of the myelin sheath, expressed as the number of myelin lamella. Axonal atrophy can be assessed in other ways. In single test fiber preparations the frequency of myelinated fibers exhibiting excessive myelin wrinkling provides a good estimate of the extent of axonal atrophy. Axonal atrophy can also be assessed by cor- 


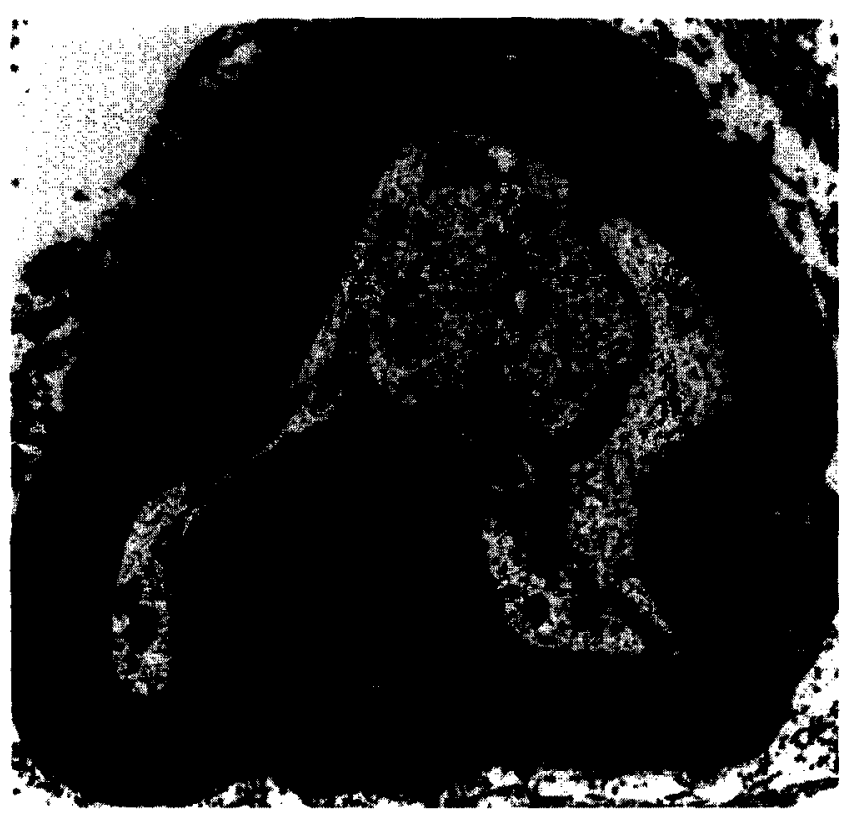

FIGURE 2 Electron micrograph of a myelinated fiber in a diabetic nerve demonstrating axonal atrophy and abnormal wrinkling of the myelin sheath.

relating the internodal length with the diameter of the fiber. Under normal circumstances, there is a linear relationship between the internodal length and the diameter of the same fiber.

The specific structural changes that occur at the node of Ranvier can be accurately quantified. For instance, axo-glial dysjunction is expressed as the number of myelin loops without junctions and is expressed as a percentage of the total number of myelin loops. When axo-glial dysjunction and nerve conduction velocity are examined in experimental diabetes in the $\mathrm{BB} / \mathrm{W}$ rat, a highly significant correlation is observed, regardless of whether the rats are normal controls, untreated diabetic rates, or treated with insulin or an aldose reductase inhibitor. ${ }^{5}$ This suggests that there is a direct correlation between this structural abnormality, axo-glial dysjunction, and the function, nerve conduction velocity.

These findings formed the basis for examinations of correlations between specific structural lesions in human diabetic nerve and nerve conduction velocity of the same nerve. These data were acquired from various clinical trials in which patients had been treated either with an aldose reductase inhibitor or a placebo.

Two abnormalities were examined: axo-glial dysjunction and axonal atrophy, which, from a theoretical viewpoint, may impact upon the propagation of the electrical impulse. These measurements were correlated with measurements of nerve conduction velocity obtained from the same sural nerves prior to biopsy. Highly significant correlations were observed between conduction velocity and the product of axoglial dysjunction and axonal atrophy, whether the latter was measured by axon/myelin ratio, by internodal diameter ratio, or by excessive wrinkling of myelinated fibers. Hence, similar to the findings in the rat model, structural abnormalities that occur in human diabetic neuropathy appear to be major determinants for the slowing of nerve conduction velocity in clinically overt diabetic neuropathy.

In a clinical trial with the aldose reductase inhibitor, sorbinil, sural nerve biopsies were obtained at the outset of the trial and following its completion. ${ }^{1}$ Sural nerve conduction velocities were obtained at the same time points. A small but significant increase in the sural nerve conduction velocity was observed in sorbinil-treated patients compared with placebo-treated patients. This was accompanied by structural improvements of axo-glial dysjunction. When axon/ myelin ratio was assessed, a significant improvement following sorbinil treatment was observed suggesting that repair of axonal atrophy occurred following treatment with sorbinil. Similarly, when internodal length/ diameter ratio or the frequency of excessively wrinkled fibers were examined, highly significant improvements occurred following sorbinil treatment. If the changes from baseline to follow-up with respect to axo-glial dysjunction and axonal atrophy were examined and compared with the change in nerve conduction velocity, highly significant correlations between the improvements in structural parameters and nerve conduction velocity were demonstrated, regardless of whether axonal atrophy was assessed by axon/myelin ratio, internodal length diameter, or by excessive myelin wrinkling (Table 1 ).

A simple and straightforward morphometric parameter for the assessment of severity of neuropathy is the myelinated fiber density. Myelinated fiber density decreases progressively with the progression of

TABLE 1. CORRELATION BETWEEN NERVE CONDUCTION VELOCITY AND MORPHOMETRIC PARAMETERS

\begin{tabular}{lcl}
\hline \multicolumn{1}{c}{ Parameter } & $r$ Value & $p$ Value \\
\hline Nerve Conduction Velocity & & \\
AGD $\times$ M/A & -0.73 & $<0.0001$ \\
AGD $\times$ IL/D & -0.65 & $<0.001$ \\
AGD $\times$ MW & -0.69 & $<0.0001$ \\
$\Delta$ Nerve Conduction Velocity & & \\
$\Delta$ AGD $\times \Delta \mathrm{M} / \mathrm{A}$ & 0.771 & $<0.001$ \\
$\Delta \mathrm{AGD} \times \Delta \mathrm{IL} / \mathrm{D}$ & 0.826 & $<0.001$ \\
$\Delta \mathrm{AGD} \times \Delta \mathrm{MW}$ & 0.860 & $<0.001$ \\
\hline
\end{tabular}

$A G D$, axo-glial dysjunction; $M / A$, myelin/axon ratio; IL/D, internodal length/fiber diameter; $M W$, myelin wrinkling; $\Delta$, change. 
the disease. We have previously shown that the amplitude of the evoked compound sensory response in diabetic nerves correlates with fiber density in the sorbinil trial. ${ }^{1}$

In another aldose reductase trial of tolrestat, conducted in patients with more severe diabetic neuropathy, an almost identical correlation between sural nerve amplitude and myelinated fiber density was demonstrated, suggesting that this is a valid structure/function correlation.

To summarize these initial findings, it can be said that

1. Sural nerve conduction velocity correlates with axo-glial dysjunction and axonal atrophy in the sural nerve.

2. Improvement in sural nerve conduction velocity following aldose reductase inhibitor treatment correlates with the improvement in axo-glial dysjunction and axonal atrophy.

3. Sural nerve compound action potential amplitude correlates well with myelinated fiber density.

\section{CORRELATION BETWEEN STRUCTURAL ABNORMALITIES AND CLINICAL ASSESSMENTS}

We have attempted to correlate the structural abnormalities occurring in sural nerve with the sensory score in patients with diabetic neuropathy. Myelinated fiber density abnormalities account for more than $20 \%$ of the defect that is seen in the sensory score. A subgroup of patients with diabetic neuropathy from two trials of aldose reductase inhibitors underwent sural nerve biopsy and were available for comparison. The two studies were the Sorbinil North American trial conducted in 28 patients, and the Tolrestat United States study in which 27 patients were studied. Sensory score was ascertained from the clinical neurologic examination. Touch, pinprick, vibration, position, and temperature sense were scored as either normal (0), reduced (1), or absent (2), and the sum of that score was used for comparison with the morphometric parameters.

In the sorbinil trial, there was a good correlation $\left(r^{2}=0.234\right)$ between myelinated fiber density and the sensory score (Figure 3). Similarly, data obtained at the termination of the tolrestat trial also revealed a high correlation $\left(r^{2}=0.44\right)$ (Figure 4). Furthermore, we correlated the same clinical parameters with a structural index of normality. The index of normality was defined as fiber density times the percentage of normal fibers assessed from teased fiber preparations. This morphometric index provides the density of structurally normal fibers in the nerve. Although the correlation was less than with density alone, significant correlations were observed between the sensory

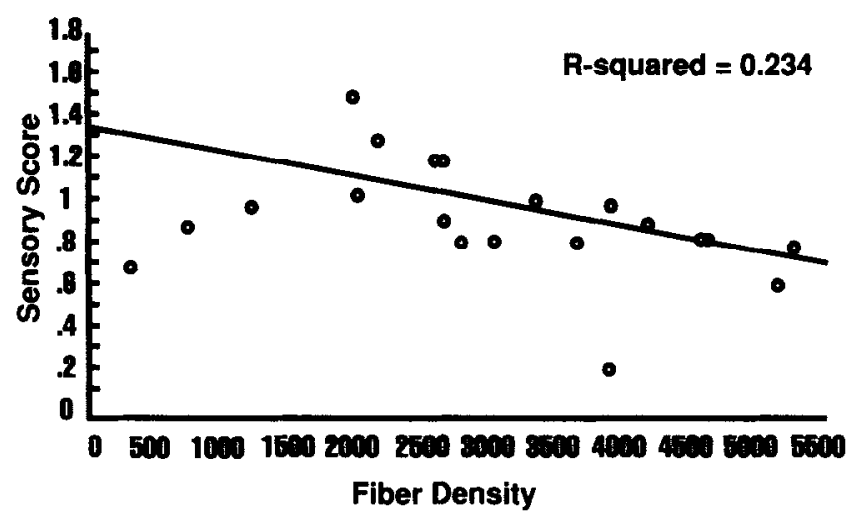

FIGURE 3 Correlation between sensory score and fiber density at the outset of the Sorbinil North American trial.

score and the index of normality in both of these trials. Therefore, correlations appear to exist between structural abnormalities and deficits obtained from a clinical neurologic examination such as the sensory score.

What is the significance of this information? Dr. Sönksen in the United Kingdom has performed power calculations of different end points to determine the number of patients required to demonstrate efficacy in a clinical trial, such as those referred to above (Table 2). If vibration threshold is used as the primary end point, the large coefficient of variation of this measure requires that an enormous number of patients be enrolled in a single trial to demonstrate significant improvements. If electrophysiologic parameters are used as primary end points, the situation is somewhat better. With motor nerve amplitude as the efficacy end point, approximately 175 patients are needed to demonstrate efficacy. With respect to the number of patients required to show a significant improvement, the best electrophysiologic parameter is nerve conduction velocity; less than 50 patients are needed in a clinical trial to show significant improvement.

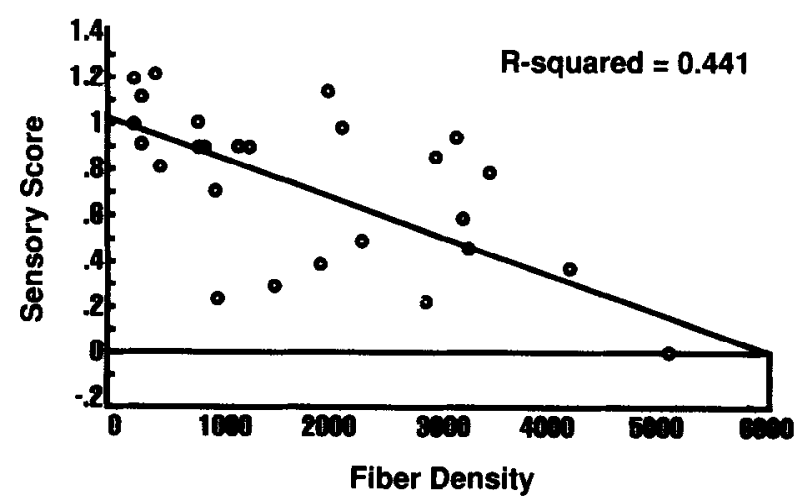

FIGURE 4 Correlation between sensory score and fiber density at the termination of the Tolrestat United States trial. 
TABLE 2. ANALYSIS OF THE NUMBER OF PATIENTS REQUIRED TO DETECT A SIGNIFICANT DIFFERENCE FOR DIFFERENT END POINTS

\begin{tabular}{lccc}
\hline \multicolumn{1}{c}{ Parameter } & $\begin{array}{c}\text { Detectable Change for } \\
\text { 55 Patients/Group; 90\% } \\
\text { Power; 5\% Significance }\end{array}$ & $\begin{array}{c}\text { Dyck and } \mathbf{O}^{\prime} \text { Brien } \\
\text { (1988) }\end{array}$ & No. of Patients/Group \\
\hline Median MNCV (m/sec) & 2.2 & 2.5 & 42 \\
Median MNA (mV) & 2.25 & 1.2 & 175 \\
Great toe VT (volts) & 6.4 & 1.4 & $>550$ \\
\hline
\end{tabular}

MNCV, motor nerve conduction velocity; MNA, motor nerve amplitude; VT, vibration threshold.

In contrast, if more precise morphometric assessment techniques are used to show significant improvement in nerve morphology, less than one-half the number of patients are required. Morphometric analyses of nerve tissue can therefore be used as sensitive efficacy end points and can be extrapolated to less precise measurements, such as electrophysiologic parameters and the sensory exam.

\section{CONCLUSIONS}

The present data suggest that in peripheral nerve specific structural, quantifiable lesions correlate closely with electrophysiologic parameters such as nerve conduction velocity and the amplitude of evoked response. Furthermore, the detailed morphometric assessment of nerve structure correlates surprisingly well with the quantified clinical exam. Due to the specificity and reproducibility of morphometric analysis, it can be used advantageously in sensitive efficacy end points in clinical neuropathy trials.

\section{REFERENCES}

1. Sima AAF, Bril V, Nathaniel V, et al: Regeneration and repair of myelinated fibers in sural nerve biopsy specimens from patients with diabetic neuropathy treated with sorbinil. N Engl J Med 319:548-555, 1988.

2. Sima AA, Lattimer SA, Yagihashi S, et al: Axo-glial dysjunction: A novel structural lesion that accounts for poorly reversible slowing of nerve conduction impulse in the spontaneously diabetic Bio-breeding rat. J Clin Invest 77:474-484, 1986.

3. Brismar T, Sima AAF: Changes in nodal function in nerve fibers of the spontaneously diabetic $\mathrm{BB} /$ Wistar rat. Acta Physiol Scand 113:499-506, 1981.

4. Sima AAF, Brismar T: Reversible diabetic nerve dysfunction: Structural correlates to electrophysiological abnormalities. Ann Neurol 18:21-29, 1985.

5. Sima AAF, Prashar A, Zhang W-X, et al: The preventive effect of long term aldose reduction inhibitors (Ponalrestat) on nerve conduction and sural nerve structure in the spontaneously diabetic BB-rat. I Clin Invest 85:1410-1420, 1990. 